\title{
Validation and reliability of the preschooler's nutrition screening tool; NutricheQ
}

\author{
H. Gibbons ${ }^{1}$, B. A. McNulty ${ }^{1}$, N. Rice ${ }^{2}$, M. J. Gibney ${ }^{1}$ and A. P. Nugent ${ }^{1}$ \\ ${ }^{1}$ UCD Institute of Food and Health, University College Dublin, Belfield, Dublin 4, Ireland and ${ }^{2}$ riceniamh@eircom.net.
}

Early identification of nutritional deficiencies through nutritional screening in children is recommended ${ }^{(1)}$. NutricheQ, developed by Danone, is a self-administered nutrition screening questionnaire for assessing nutritional risk in preschool children. It comprises of 18 questions divided into 3 sections with section 1 and 2 generating the questionnaire's total risk score and a higher score indicating increased nutritional risk.

This study tested the reliability of NutricheQ and aimed to validate the ability of the questionnaire to identify children at risk using data from the Irish National Preschool Nutrition Survey (NPNS) ${ }^{(2)}$ which recorded food and beverage consumption in a representative sample of Irish preschoolers. Principle component analysis (PCA) was used in conjunction with Cronbach's alpha, as a test of reliability. Validation methods included the examination of mean daily intakes across quartiles of the NutricheQ score with the highest NutricheQ scores indicating high risk (Quartile 4) and the lowest NutricheQ scores indicating low risk (Quartile 1).

\begin{tabular}{|c|c|c|c|c|c|c|c|c|}
\hline \multirow{5}{*}{$\begin{array}{l}\text { NutricheQ Score } \\
\text { N }\end{array}$} & \multicolumn{8}{|c|}{ Quartiles of NutricheQ score } \\
\hline & \multicolumn{2}{|c|}{ Quartile 1} & \multicolumn{2}{|c|}{ Quartile 2 } & \multicolumn{2}{|c|}{ Quartile 3 } & \multicolumn{2}{|c|}{ Quartile 4} \\
\hline & \multicolumn{2}{|c|}{$0-3$} & \multicolumn{2}{|c|}{$4-5$} & \multicolumn{2}{|c|}{$6-7$} & \multicolumn{2}{|c|}{$8-13$} \\
\hline & \multicolumn{2}{|c|}{74} & \multicolumn{2}{|c|}{104} & \multicolumn{2}{|c|}{96} & \multicolumn{2}{|c|}{87} \\
\hline & Mean & (SD) & Mean & (SD) & Mean & (SD) & Mean & (SD) \\
\hline$\overline{\text { Age (yrs) }}$ & $1.7^{\mathrm{a}}$ & 0.8 & $1.9^{\mathrm{a}}$ & 0.8 & $2.0^{\mathrm{a}}$ & 0.8 & $2.4^{\mathrm{b}}$ & 0.7 \\
\hline Energy (MJ) & 4.6 & 1.1 & 4.6 & 1.1 & 4.5 & 1.0 & 4.6 & 1.0 \\
\hline Protein (\%TE) & $16.0^{\mathrm{a}}$ & 2.8 & $15.4^{\mathrm{ab}}$ & 2.4 & $15.2^{\mathrm{ab}}$ & 2.0 & $14.5^{\mathrm{b}}$ & 2.5 \\
\hline Dietary Fibre (g/10MJ) & $27.7^{\mathrm{a}}$ & 6.7 & $25.0^{\mathrm{b}}$ & 7.2 & $24.7^{\mathrm{b}}$ & 6.6 & $21.4^{\mathrm{c}}$ & 6.8 \\
\hline Sat fat (\%TE) & 15.9 & 3.4 & 15.8 & 3.6 & 15.4 & 3.4 & 15.6 & 3.7 \\
\hline NMS (\%TE) & $14.0^{\mathrm{a}}$ & 5.1 & $16.1^{\mathrm{ab}}$ & 5.2 & $17.1^{\mathrm{b}}$ & 6.3 & $17.0^{\mathrm{ab}}$ & 7.0 \\
\hline Iron $(\mathrm{mg} / 10 \mathrm{MJ})$ & $18.0^{\mathrm{a}}$ & 6.0 & $16.3^{\mathrm{ab}}$ & 5.6 & $15.1^{\mathrm{bc}}$ & 5.6 & $13.3^{\mathrm{c}}$ & 4.4 \\
\hline Vitamin D $(\mu \mathrm{g} / 10 \mathrm{MJ})$ & $8.4^{\mathrm{a}}$ & 7.8 & $6.4^{\mathrm{ab}}$ & 6.9 & $5.2^{\mathrm{b}}$ & 6.0 & $4.4^{\mathrm{b}}$ & 5.1 \\
\hline Zinc $(\mathrm{mg} / 10 \mathrm{MJ})$ & $13.1^{\mathrm{a}}$ & 3.4 & $11.6^{\mathrm{b}}$ & 2.6 & $10.9^{\mathrm{bc}}$ & 2.7 & $10.0^{\mathrm{c}}$ & 2.3 \\
\hline Calcium (mg/10MJ) & $1935.1^{\mathrm{a}}$ & 513.3 & $1771.1^{\mathrm{ab}}$ & 503.0 & $1641.9^{\mathrm{b}}$ & 512.4 & $1561.6^{\mathrm{ab}}$ & 571.8 \\
\hline Sodium (mg/10MJ) & 2390.3 & 680.8 & 2304.9 & 644.8 & 2506.4 & 623.4 & 2583.3 & 645.0 \\
\hline Riboflavin (mg/10MJ) & $3.6^{\mathrm{a}}$ & 1.0 & $3.4^{\mathrm{a}}$ & 1.0 & $3.2^{\mathrm{ab}}$ & 0.9 & $2.9^{\mathrm{b}}$ & 1.0 \\
\hline Niacin $(\mathrm{mg} / 10 \mathrm{MJ})$ & $24.5^{\mathrm{a}}$ & 6.1 & $24.3^{\mathrm{a}}$ & 8.4 & $23.1^{\mathrm{ab}}$ & 6.5 & $21.5^{\mathrm{b}}$ & 7.2 \\
\hline Folate $(\mu \mathrm{g} / 10 \mathrm{MJ})$ & $411.1^{\mathrm{a}}$ & 169.3 & $382.0^{\mathrm{ab}}$ & 154.7 & $343.6^{\mathrm{b}}$ & 118.7 & $340.8^{\mathrm{b}}$ & 171.4 \\
\hline Phosphorous(mg/10MJ) & $1917.7^{\mathrm{a}}$ & 359.7 & $1845.1^{\mathrm{a}}$ & 335.8 & $1786.0^{\mathrm{ab}}$ & 330.2 & $1696.1^{\mathrm{b}}$ & 359.3 \\
\hline Potassium (mg/10MJ) & $3950.1^{\mathrm{a}}$ & 613.0 & $3976.0^{\mathrm{a}}$ & 656.9 & $3772.5^{\mathrm{ab}}$ & 631.3 & $3476.7^{b}$ & 744.0 \\
\hline Carotene $(\mu \mathrm{g} / 10 \mathrm{MJ})$ & $6022.3^{\mathrm{a}}$ & 6329.4 & $5179.7^{\mathrm{ab}}$ & 3711.2 & $3800.9^{\mathrm{bc}}$ & 3900.4 & $2631.8^{\mathrm{c}}$ & 2877.2 \\
\hline Total Fruit (g) & $138.6^{\mathrm{a}}$ & 70.6 & $144.8^{\mathrm{a}}$ & 91.3 & $140.9^{\mathrm{a}}$ & 83.6 & $96.2^{\mathrm{b}}$ & 82.8 \\
\hline Total Vegetables (g) & $77.8^{\mathrm{a}}$ & 45.7 & $62.1^{\mathrm{b}}$ & 34.9 & $50.9^{\mathrm{bc}}$ & 29.2 & $37.7^{\mathrm{c}}$ & 28.4 \\
\hline
\end{tabular}

$\% \mathrm{TE}=$ Percentage contribution to total energy intake. NMS = Non Milk Sugars. ${ }^{\text {abcd }}$ Different superscript letters indicate significance between quartiles (One-Way Analysis of

Covariance followed by Bonferroni's test). Values not showing common significance $(p<0.05)$.

PCA and cronbach's alpha revealed NutricheQ to be a multidimensional questionnaire with reasonable reliability (0.447). Across the quartiles of risk score, children who had a higher risk score (Quartile 4) had significantly lower protein, fibre, iron, vitamin D, zinc, riboflavin, niacin, folate, phosphorous, potassium, carotene and fruit and vegetable intakes compared to children with lower risk scores (Quartile 1). A greater proportion of children in quartile 4 tended to have intakes of iron, vitamin A and zinc below recommendations compared to children in quartile 1.

This data suggests that NutricheQ is a reliable and valid multi-dimensional nutritional screening questionnaire. It successfully identified toddlers with poorer nutritional intakes. However, further refinements could improve reliability and consider differences in food intakes.

This analysis was supported by Danone Trading B.V. The NPNS was funded by the Irish Department of Agriculture, Fisheries and Food under the Food for Health Research Initiative (2007-2012).

1. Randall Simpson JA, et al. (2008) Eur J of Clin Nutr 62(6), 770-780.

2. Irish Universities Nutrition Alliance (2012) The National Preschool Nutrition Survey. http://www.iuna.net/ 